\title{
Construct validity and internal consistency of the Breast Inflammatory Symptom Severity Index in lactating mothers with inflammatory breast conditions
}

\author{
Emma Heron ${ }^{\text {Corresp., } 1}$, Adelle McArdle $^{2}$, Md Nazmul Karim ${ }^{3}$, Melinda Cooper ${ }^{4}$, Donna Geddes ${ }^{5}$, Leanda McKenna ${ }^{1}$ \\ ${ }^{1}$ School of Allied Health, Curtin University, Bentley, Western Australia, Australia \\ 2 Monash Rural Health, Monash University, Churchill, Victoria, Australia \\ ${ }^{3}$ School of Public Health and Preventative Medicine, Monash University, Melbourne, Victoria, Australia \\ 4 MMC Physiotherapy, Kyneton, Victoria, Australia \\ 5 School of Molecular Sciences, University of Western Australia, Crawley, Western Australia, Australia \\ Corresponding Author: Emma Heron \\ Email address: emma.duff@postgrad.curtin.edu.au
}

Background. Inflammatory Conditions of the Lactating Breast (ICLB) affect more than one in five lactating mothers, yet no fully validated outcome measures exist to aid clinicians in their patient-centred care of women with ICLB. The Breast Inflammatory Symptom Severity Index (BISSI) is an ICLB-specific clinician administered patient-reported outcome measure, currently used by Australian clinicians, who treat mothers with ICLB. To date the BISSI has undergone partial psychometric development. This study, therefore, aimed to undertake the next stage of psychometric development by determining the construct validity and internal consistency of the BISSI.

Methods. A retrospective audit was conducted on patient records of 160 mothers who were treated for ICLB, at a private physiotherapy practice in Melbourne, Australia. An electronic data capture tool was used to collate BISSI scores and associated ICLB assessment variables. Construct validity was determined through factor analysis and discriminant performance. Reliability was determined by assessing measures of internal consistency.

Results. Factor analysis established that BISSI items $(n=10)$ loaded on to four factors, Wellness, Pain, Physical Characteristics of Affected Area (PCAA), and Inflammation, which together, explained $71.2 \%$ of variance. The remaining item ('Wellness/sickness unspecified') did not load. Wellness, Pain, PCAA and Inflammation factors individually and collectively displayed the ability to discriminate symptom severity, as scores were significantly higher in mothers with high symptom severity (assessed via AUC close to or > 0.7 and $P$ value $<0.005$ for each factor). The BISSI demonstrated internal consistency with an overall Cronbach alpha of 0.742 . 
Conclusions. The BISSI has adequate construct validity, demonstrating behaviour consistent with theoretical constructs of inflammation severity, via its dimensionality and ability to discriminate symptom severity. The BISSI also has adequate internal consistency demonstrating reliability. Therefore, clinicians can have confidence that the BISSI is valid, the individual item scores are correlated, and the concepts are consistently measured. 
1 Construct validity and internal consistency of the Breast Inflammatory Symptom Severity

2 Index in lactating mothers with inflammatory breast conditions

3 Emma Louise Heron ${ }^{1}$, Adelle Margaret McArdle ${ }^{2}$, Md Nazmul Karim ${ }^{3}$, Melinda Mary Cooper 4 ,

4 Donna Tracy Geddes ${ }^{5}$ \& Leanda Jane McKenna ${ }^{1}$

$5 \quad{ }^{1}$ School of Allied Health, Curtin University, Bentley, Western Australia, Australia

$6 \quad{ }^{2}$ Monash Rural Health, Monash University, Churchill, Victoria, Australia

$7{ }^{3}$ School of Public Health and Preventive Medicine, Monash University, Melbourne, Victoria,

8 Australia

$9 \quad{ }^{4}$ MMC Physiotherapy, Kyneton, Victoria, Australia

$10{ }^{5}$ School of Molecular Sciences, University of Western Australia, Crawley, Western Australia,

11 Australia

12 Corresponding author:

13 Emma Heron ${ }^{1}$

14 Email address: emma.duff@postgrad.curtin.edu.au 


\section{Abstract:}

Background. Inflammatory Conditions of the Lactating Breast (ICLB) affect more than one in five lactating mothers, yet no fully validated outcome measures exist to aid clinicians in their patient-centred care of women with ICLB. The Breast Inflammatory Symptom Severity Index (BISSI) is an ICLB-specific clinician administered patient-reported outcome measure, currently used by Australian clinicians, who treat mothers with ICLB. To date the BISSI has undergone partial psychometric development. This study, therefore, aimed to undertake the next stage of psychometric development by determining the construct validity and internal consistency of the BISSI.

Methods. A retrospective audit was conducted on patient records of 160 mothers who were treated for ICLB, at a private physiotherapy practice in Melbourne, Australia. An electronic data capture tool was used to collate BISSI scores and associated ICLB assessment variables.

Construct validity was determined through factor analysis and discriminant performance.

Reliability was determined by assessing measures of internal consistency.

Results. Factor analysis established that BISSI items $(n=10)$ loaded on to four factors, Wellness, Pain, Physical Characteristics of Affected Area (PCAA), and Inflammation, which together, explained $71.2 \%$ of variance. The remaining item ('Wellness/sickness unspecified') did not load. Wellness, Pain, PCAA and Inflammation factors individually and collectively displayed the ability to discriminate symptom severity, as scores were significantly higher in mothers with high symptom severity (assessed via AUC close to or $>0.7$ and $\mathrm{P}$ value $<0.005$ for each factor). The BISSI demonstrated internal consistency with an overall Cronbach alpha of 0.742 . 
36 Conclusions. The BISSI has adequate construct validity, demonstrating behaviour consistent

37 with theoretical constructs of inflammation severity, via its dimensionality and ability to

38 discriminate symptom severity. The BISSI also has adequate internal consistency demonstrating

39 reliability. Therefore, clinicians can have confidence that the BISSI is valid, the individual item

40 scores are correlated, and the concepts are consistently measured. 
41

42 Inflammatory conditions of the lactating breast (ICLB) include non-physiological engorgement,

43 blocked ducts, mastitis, and breast abscess (Heron et al. 2020). These conditions are all

44

characterised by a combination of local, and/or systemic signs of inflammation such as breast pain, redness, swelling, heat, and fever and flu-like symptoms (LactaResearch Group 2018).

Clinical presentations of ICLB vary greatly, from a few local breast symptoms to rapid onset of acute physical illness, that may substantially interfere with a mother's physical and emotional daily functioning (Amir \& Lumley 2006). The exact aetiology and role of bacterial pathogens in the clinical manifestations of ICLB is unclear and has been debated over the last three decades (Collado et al. 2009; Ingman et al. 2014; Kvist et al. 2008). Scientific evidence now suggests that bacterial species may not be the primary causative agents, and rather, ICLB may be the result of a transient alteration of the mother's milk microbiome, along with genetic and environmental factors (Delgado et al. 2008; Hunt et al. 2011; Ingman et al. 2014; Jahanfar et al. 2013; LaTuga et al. 2014). A recent systematic review (Wilson et al. 2020) found that approximately one in four lactating mothers are affected by mastitis during the first six months postpartum. Consequently, many mothers present to clinicians in the early postpartum period, seeking relief for their often debilitating symptoms of breast inflammation.

In Australia, mothers with ICLB commonly seek treatment from general practitioners, lactation consultants, general and women's health physiotherapists, and hospital emergency departments. Each profession provides different treatments for ICLB, although their roles may overlap. Common treatments for ICLB provided by Australian physiotherapists include therapeutic ultrasound, education and advice, massage, Tubigrip ${ }^{\circledR}$, kinesiology tape and low-level laser therapy (Diepeveen et al. 2019). The absence of a fully validated clinical measure for ICLB 
64 limits clinicians' capacity to appropriately assess mothers, monitor their treatment response, and

65 follow their clinical progress over the course of their condition (Kyte et al. 2014). Current

66 clinical practice is comprised of subjective assessment of symptoms, which forms the basis for

67 diagnostic and treatment decision making (Amir et al. 2014). Therefore, a psychometrically

68 robust tool to measure the most important clinical presentations of ICLB is required. Accurate

69 assessment of the cardinal signs and symptoms of inflammation (pain, redness, swelling, heat

70 and loss of function) (Scott et al. 2004) and the changes in these inflammatory symptoms that

71 may be attributed to treatment will aid in the care for women with ICLB. Validated outcome

72 measures for use in ICLB interventional clinical trials, are also required to help develop high-

73 level evidence for ICLB treatments. Such a tool would also be useful to improve and provide

74 impetus for future international research on ICLB.

75 The Breast Inflammatory Symptom Severity Index (BISSI) is an ICLB-specific Clinician

76 Administered Patient Reported Outcome Measure (CAPROM) (Cooper et al. 2020). It is

77 currently in clinical use by clinicians who have attended the Australian 'Lactation for Health

78 Professionals' course (Inform Physiotherapy 2021). The BISSI was originally developed to

79 provide clinicians and mothers with a simple, quick, immediate, and prognostic measure of

80 symptom severity, capturing all the inflammatory symptoms. It is administered at the time of

81 consultation and uses a Numerical Rating Scale (NRS) to measure pain, systemic symptoms, and

82 functional impact; and a 5-point scale to measure breast hardness/tightness (swelling), breast

83 temperature, redness, and size of the affected area (see Table 1). An accompanying clinician

84 script was developed to preserve face validity and utility (Cooper et al. 2020). Ease of utility was

85 deemed particularly important, since mothers with ICLB can be acutely unwell and clinicians

86 administering the tool have varied experience with ICLB (Cooper et al. 2020). The BISSI is the 
87 only ICLB PROM to have undergone partial psychometric development. While its face and

88 content validity have been recently established (Cooper et al. 2020), further psychometric

89 evaluation is required.

90 The aim of this study was to determine the psychometric properties of the BISSI to further

91 develop the tool for use in clinical settings and ICLB efficacy trials (Cooper et al. 2020).

92 Specifically, an exploration of construct validity, via an examination of the dimensionality and ability to discriminate severity of symptoms was undertaken. An assessment of the reliability of

94 the BISSI, through an examination of internal consistency, was also undertaken.

\section{Materials \& methods}

A retrospective audit of patient clinical notes of mothers with ICLB was performed at a private physiotherapy practice in Melbourne, Australia. Ethical approval for this study was granted by Curtin University Human Research Ethics Committee (HRE2020-0544) which included a waiver of consent.

101

102

103

104

105

106

107

108

\section{Participants/Sample}

Clinical appointment notes from 160 lactating mothers who presented to the private physiotherapy practice between 12 July 2017 and 15 September 2020 with an ICLB, were examined (Fig. 1). Clinical notes from an appointment were considered eligible if the mother had presented with ICLB, was over 18 years of age and had an accompanying completed record of their BISSI scores at their initial appointment. The initial appointment was defined as the first appointment or contact with the clinician for care of a defined episode of ICLB. An ICLB episode was the period from initial onset of ICLB symptoms until complete resolution. Data for 
109 one ICLB episode per mother was collected. The practice management software (Cliniko,

110 Melbourne, Australia) (Cliniko 2010), was used to identify and scrutinise eligible clinical notes.

111 A search of all ICLB appointment types (initial, review and extended) was conducted in Cliniko

112 from 15 September 2020 until 160 eligible case notes were identified. The auditor (EH), an

113 experienced Women's Health physiotherapist registered with Australian Health Practitioner

114 Regulation Agency, used the Cliniko appointment diary to search on a day-by-day basis, with the

115 'hide names' function activated to maintain anonymity of non-ICLB patients. Appointment types

116 were colour-coded in Cliniko, hence ICLB appointments could be identified. An episode was

117 identified by locating an initial appointment or clear documentation of a new ICLB episode

118 within the clinical record. Where multiple episodes of care occurred, only the data from the most

119 recent ICLB episode was used.

120 The BISSI has 11 assessment items (see Table 1), producing eight individual item scores. The

121 statistical analysis required 10 to 20 scores per item to produce stable factor analysis solutions

122 with reduced sampling error (Maccallum et al. 1999; Thompson 2004). Therefore, based on the

123 sample size recommendations in factor analysis, 160 complete BISSI scores or eligible clinical

124 notes were examined, derived from eight scores multiplied by 20.

\section{Procedure}

126 Data collection was performed in October 2020. Research Electronic Data Capture, a secure, 127 web-based software platform designed to support data capture for research studies, hosted at 128 Curtin University, was used to collect, and manage the data (Harris et al. 2019; Harris et al. 129 2009; REDCap 2004). Forced response and validation rules were used, to minimise data 130 omission errors. Three data collection variable domains were created. The first domain 
131 comprised demographic data of maternal date of birth, maternal parity, breastfeeding infant's

132 age, mode of delivery, single/multiple birth, month of presentation and socioeconomic status,

133 including postcode, private health insurance and maternal occupation. With respect to mother's

134 occupation, an Occupational Socioeconomic Status Scale, modified from Marks (2000) (Marks

135 et al. 2000), was used to classify occupational data (see Table S1). The scale consists of six

136 groups, with four hierarchical occupation levels (group 1 to 4 respectively) based on required

137 skill level and skill specialisation. Group 5 represented those not currently in paid work, and

138 group 6 represented occupation unreported. Three members of the research team (EH, AM, LM)

139 independently used the scale to classify 66 different occupations named by the mothers. Where

140 discrepancies (29) occurred, majority consensus was used to assign a score. For the postcode

141 socioeconomic status measure, the Australian Bureau of Statistics, Socio-Economic Indexes for

142 Areas 2016, Index of Relative Socio-Economic Advantage and Disadvantage was used. Within

143 the index, the postal area decile ranking within Australia was used, which ranks postal area codes

144 from lowest to highest advantage, with a decile number of 1 representing the lowest $10 \%$ of

145 postal areas (most disadvantaged) up to a decile number of 10 , the highest $10 \%$ of postal areas

146 (most advantaged).

147 The second domain of ICLB characteristics comprised the affected breast (right or left) and

148 quadrant, number and type of local and systemic symptom(s), including symptom onset, and

149 antibiotic use. The third domain of clinician assessment comprised BISSI scores and clinician

150 breast and nipple observation (including number and type of local breast inflammatory

151 symptoms). 


\section{Analysis}

153 Pre-analysis data screening demonstrated no violations to the assumptions of linearity, normality,

154 multicollinearity, and homoscedasticity. All individual item scales were standardised for

155 maximal scale length for the analysis, given the heterogeneity across the BISSI items (see Table

156 1). Standardisation occurred by converting all Likert scales into the 11-point NRS scale (0-10),

157 similar to what is used to measure pain (Karcioglu et al. 2018), creating scale homogeneity. This

158 ensured individual items contributed equal weight to the total score.

159 Descriptive statistics were generated for maternal demographics and characteristics, and BISSI

160 items. Exploratory Factor Analysis (EFA) using Principal Component Analysis (PCA) was used

161 to identify underlying factors within the BISSI (Field 2009; Streiner 2015). Confirmatory Factor

162 Analysis (CFA) cross-validated the factor structure derived from EFA, to establish the

163 dimensionality of the BISSI. Items of the BISSI that loaded onto the same factors were combined

164 as a 'factor' and factor-specific validity and reliability was assessed subsequently. Individual

165 items were screened to identify those with poor factor loading $(<0.3)$.

Discriminant validity was explored by assessing the BISSI's ability to discriminate between mothers with high and low symptom severity. Mothers with mastitis were considered likely to have the highest scores or severity. Other conditions within the suite of ICLB are not considered to be as severe (Betzold 2007), providing a suitable differentiation against mastitis for discriminant analysis. A diagnosis of mastitis (Amir \& Academy of Breastfeeding Medicine Protocol Committee 2014; Amir et al. 2014), as being “... at least 2 breast signs/symptoms (pain, lump/hardness, redness) and fever or at least 1 systemic symptom (lethargy, aching, headache, nausea and so on)", determined mothers of high symptom severity. 
174 Reliability (measured using internal consistency) of the BISSI was assessed using Cronbach's $\alpha$ 175 coefficient (Field 2009). A Cronbach alpha value of 0.70 or above was considered an acceptable 176 level of internal consistency (Taber 2018). The contribution of each item and factor on the BISSI

177 was assessed by generating the item-total and factor-total correlation and by computing 178 Cronbach's alpha excluding that item or factor, respectively.

179 All statistical analyses were performed using statistical software R-3.6.0 (The R Project for 180 Statistical Computing) (R Core Team 2017), and STATA/IC release 15 (StataCorp 2017) where 181 appropriate.

\section{Results}

\section{Demographics}

185 A total of 290 ICLB clinical notes from 197 mothers were identified initially and examined for eligibility (Fig. 1). There were 21 mothers with ineligible clinical notes, due to no or incomplete initial appointment BISSI scores, and 16 mothers who had more than one identified ICLB episode during the collection period. For these 16 mothers, their most recent ICLB episode case notes were included in this study and prior episodes were excluded.

Demographics for the 160 included mothers are presented in Table 2. In general, mothers were aged in their thirties and their breastfeeding infants ranged from 4 days to 21 months old. Most mothers had a high socioeconomic status, as they lived in the most advantaged postal areas, had private health insurance, and were employed in professional occupations. In unilateral ICLB presentations, the right breast was most affected, and the most common location on the right 
195 breast was the inferior lateral quadrant (see Fig. 2). Nearly half the mothers matched the criteria 196 (Amir et al. 2014) for diagnosis of mastitis during their current ICLB episode.

\section{Construct validity}

\section{Factor analysis}

199 Preliminary exploratory factor analysis (EFA) revealed the item 'Wellness/sickness unspecified' 200 had a factor loading of less than 0.3. Confirmatory factor analysis extracted four distinct factors, 201 Pain (incorporating items Pain Awareness and Pain Touch), Wellness (incorporating items 202 Fever, Ache, Headache), Physical Characteristics of Affected Area (PCAA) (incorporating items 203 Hardness, Area, Impact) and Inflammation (incorporating items Redness and Temperature) (Fig. 2043 , see correlations in Table 3). The four factors together explained $71.2 \%$ of the variation in the 205 score (Table 3).

\section{Discriminant validity}

The BISSI and all its factor scores were found to be significantly higher in mothers with high symptom severity (Table 4). The Area under the Curve (AUC) analysis (factors of Wellness,

Pain, Inflammation, PCAA and BISSI), indicated the BISSI can correctly identify high symptom

210 severity in close to or greater than $70 \%$ of the participants (Table 5). The Receiver Operating

211 Characteristic (ROC) analysis (Fig. 4) indicates a good level of discriminatory accuracy for the

212 BISSI and all factors.

\section{Internal consistency}

214 The BISSI showed high internal consistency (Table 6). The overall Cronbach's $\alpha$ coefficient for 215 the BISSI was above 0.7 indicating the BISSI was reliable and repeatable (Table 6). Item-total 216 correlation across items ranged from 0.25 to 0.56 (Table 7). All the items either caused a 
217 decrease or no change in the overall BISSI Cronbach's $\alpha$ values upon removal from the BISSI,

218 except for the items Fever, Hardness, and Redness (Table 7).

219 All factors showed internal consistency with individual factor Cronbach's $\alpha$ values for Wellness,

220 Pain, Inflammation and BISSI (total score) factors above the acceptable 0.7 Cronbach's $\alpha$ value

221 for internal consistency (Table 6). The Cronbach's $\alpha$ value for the factor of PCAA (incorporating

222 items Hardness, Area, and Impact) was just under 0.7 (Table 6).

Discussion

225 This retrospective audit of clinical notes from mothers with ICLB, provides evidence supporting the validity and reliability of the BISSI. Discriminant validity and internal consistency were established, demonstrating the ability of the BISSI to discriminate and reliably measure symptom severity in mothers with ICLB. Overall, the clinical tool performed well psychometrically.

The factor analysis revealed a four-factor structure underlying 10 of the 11 items on the BISSI, with one item, 'Wellness/Sickness unspecified', not contributing to the factor structure. This item originally allowed mothers to rate their degree of systemic 'wellness or sickness' without specifying symptoms, if they did not have the already specified symptoms of fever, generalised aches and pains, or headache. Measuring a mother's 'worst' sickness using the

'Wellness/Sickness unspecified' item was found to be imprecise when measuring ICLB symptoms, presumably because many reasons can influence a mother's state of wellness.

236 Therefore, it is proposed that the item 'Wellness/Sickness unspecified' be removed from the 237 BISSI.

238 The three specific systemic symptoms of fever, generalised aches and pains and headache, strongly loaded on to the factor 'Wellness'. These findings align with mothers' reports of ICLB, 
240 which commonly include the presence of flu-like symptoms, such as fever, aches and pains,

241 and/or headache (Cooper et al. 2020; Heron et al. 2020; Kvist 2006). The BISSI asks mothers to

242 select their worst symptom of the three and rate the severity of this symptom only. However, the

243 mother's selected symptom can differ on subsequent BISSI responses. Therefore, it is proposed

244 that these three symptoms of fever, aches and pains, and headache, are all included within the

245 BISSI item wording, to give one all-encompassing severity rating for these ICLB systemic 246 symptoms.

247 The BISSI items of Impact, Affected Area and Hardness/Tightness loaded on to the factor 248 'PCAA', indicating a correlation between the severity, size of affected area, swelling or 249 hardness/tightness, and impact/interference on the mother's everyday life. This loading is 250 consistent with inflammation theory which includes the fifth cardinal sign and symptom, loss of 251 function (Scott et al. 2004). The BISSI is therefore measuring the important cardinal signs and 252 symptoms of inflammation, and no change is proposed to these items on the BISSI as a result of 253 these findings.

254 As both pain items (awareness and touch) loaded strongly on to the factor 'Pain', we propose 255 incorporating both pain items in the final index score of the BISSI. It may be important to 256 maintain the separate distinction of pain on 'awareness' versus 'touch' by incorporating both in 257 the final score, as pain on awareness may be a manifestation of peripheral sensitisation.

258 Additionally, a high score against 'pain awareness' may indicate the condition is developing 259 central modulation (Baron et al. 2013). This change is supported by prior findings, whereby pain was rated as the most important symptom in ICLB, and the symptom perceived to change the 261 fastest in response to treatment (Cooper et al. 2020). Importantly, all proposed changes align with previous research, wherein mothers indicated that the tool must capture their range and 
263 severity of symptoms and concerns, while also being concise and accurate (Cooper et al. 2020).

264 The BISSI may help the clinician to determine if ICLB severity is worsening or abating and thus

265 whether treatments are effective. The final proposed change to the BISSI includes scoring all

266 items similarly using an NRS. This was a change implemented for statistical analysis conducted

267 in this study, to ensure equal weighting of individual items in the BISSI.

268 The BISSI demonstrated good construct validity and can discriminate based on symptom

269 severity. Clinically, this shows the BISSI can distinguish between mothers with high and low

270 ICLB symptom severity. This means clinicians can have greater confidence that the BISSI

271 measures the theoretical construct of symptom severity. The BISSI also demonstrated internal

272 consistency, indicating it is a reliable measure and clinicians can have confidence that the items

273 reliably measure a similar construct.

274 This is the second study to have measured psychometric properties of the BISSI, providing

275 another important step towards the development of a psychometrically robust ICLB-specific

276 CAPROM for clinicians and researchers. Further psychometric testing of the BISSI is required to

277 prospectively assess the tool's responsiveness to change over time and determine its' convergent

278 and criterion validity. Further measurement and analysis of the BISSI should aim to determine

279 the weighting of individual items along with the contribution of each item to the total severity

280 index score. Future analysis of the BISSI would help ensure standardised clinical data is

281 recorded, alongside accepted criterion outcome measures.

282 While a strength of this study is its sample size, it is limited by its retrospective cross-sectional

283 design and the potential for selection bias. Mothers were selected from one private physiotherapy

284 practice, and most were of high socioeconomic status, and may not be representative of all

285 mothers with ICLB. Additionally, different clinicians were often involved in the mother's care, 
286 as ICLB requires scheduling appointments as soon as possible with the clinician available at the 287 time. This may have contributed to inconsistencies in documentation and outcome measurement 288 assessment, potentially imposing additional limitations. Further, the extent of information shared 289 by the patient and documented by the clinician may have been limited, to prioritise safe, timely 290 treatment formulation and delivery. Thus, if a symptom was not documented, it could not be 291 assumed it was not present. Yet, another strength of this study is its contribution to producing an 292 internally valid and discriminatory CAPROM, which will benefit further prospective studies and 293 enhance clinician assessment and treatment of ICLB. There is currently no gold standard 294 outcome measure for ICLB, which has impeded assessment of criterion validity.

\section{Conclusions}

297 This study provides evidence for the construct validity of the BISSI by establishing its ability to 298 discriminate the severity of inflammatory breast symptoms. Its internal consistency reliability 299 was also established. Changes to the tool are proposed to provide an updated outcome measure 300 for clinical use and prepare the BISSI for further psychometric evaluation in future studies.

301 Clinicians should be trained to use the BISSI, to ensure consistent and accurate utility of this 302 ICLB-specific CAPROM. 
303

304

305

306

307

308

309

310

311

312

313

314

315

316

317

318

319

320

321

322

323

324

325

326

327

328

329

330

331

332

333

334

335

336

337

338

339

340

341

342

343

344

\section{Acknowledgements}

We wish to acknowledge the private physiotherapy practice involved in the audit, and thank the

practice's director, Hillary Schwantzer, for enabling this research.

\section{References}

Amir LH, and Academy of Breastfeeding Medicine Protocol Committee. 2014. ABM clinical protocol \#4: Mastitis, revised March 2014. Breastfeed Med 9:239-243. $10.1089 / \mathrm{bfm} .2014 .9984$

Amir LH, and Lumley J. 2006. Women's experience of lactational mastitis: 'I have never felt worse'. Aust Fam Physician 35:745-747.

Amir LH, Trupin S, and Kvist LJ. 2014. Diagnosis and treatment of mastitis in breastfeeding women. J Hum Lact 30:10-13. 10.1177/0890334413516065

Baron R, Hans G, and Dickenson AH. 2013. Peripheral input and its importance for central sensitization. Ann Neurol 74:630-636. 10.1002/ana.24017

Betzold CM. 2007. An update on the recognition and management of lactational breast inflammation. J Midwifery Womens Health 52:595-605. 10.1016/j.jmwh.2007.08.002

Cliniko. 2010. Cliniko: Allied Health Practice Management Software. Available at https://www.cliniko.com/ (accessed 10 Jan 2021).

Collado MC, Delgado S, Maldonado A, and Rodríguez JM. 2009. Assessment of the bacterial diversity of breast milk of healthy women by quantitative real-time PCR. Letters in Applied Microbiology 48:523-528. https://doi.org/10.1111/j.1472-765X.2009.02567.x

Cooper M, Lowe H, and McArdle A. 2020. Development of a novel patient focussed symptom severity index for use in assessing and treating inflammatory conditions of the lactating breast: a Delphi study. International journal of evidence-based healthcare. 10.1097/XEB.0000000000000225

Delgado S, Arroyo R, Martín R, and Rodríguez JM. 2008. PCR-DGGE assessment of the bacterial diversity of breast milk in women with lactational infectious mastitis. $B M C$ Infect Dis 8:51-51. 10.1186/1471-2334-8-51

Diepeveen LC, Fraser E, Croft AJE, Jacques A, McArdle AM, Briffa K, and McKenna L. 2019. Regional and Facility Differences in Interventions for Mastitis by Australian Physiotherapists. J Hum Lact 35:695-705. 10.1177/0890334418812041

Field AP. 2009. Chapter 15: Exploratory Factor Analysis Discovering statistics using SPSS (and sex and drugs and rock ' $n$ ' roll). 3rd ed. London: London : SAGE, 619-679.

Harris PA, Taylor R, Minor BL, Elliott V, Fernandez M, O'Neal L, McLeod L, Delacqua G, Delacqua F, Kirby J, and Duda SN. 2019. The REDCap consortium: Building an international community of software platform partners. Journal of Biomedical Informatics 95:103208. https://doi.org/10.1016/j.jbi.2019.103208

Harris PA, Taylor R, Thielke R, Payne J, Gonzalez N, and Conde JG. 2009. Research electronic data capture (REDCap) - A metadata-driven methodology and workflow process for providing translational research informatics support. Journal of Biomedical Informatics 42:377-381. https://doi.org/10.1016/j.jbi.2008.08.010

Heron E, Maselli T, McArdle A, De Oliveira B, and McKenna L. 2020. Exploring physiotherapists' clinical definition and diagnosis of inflammatory conditions of the 
lactating breast in Australia: a mixed methods study. International Breastfeeding Journal 15. 10.1186/s13006-020-00294-9

Hunt KM, Foster JA, Forney LJ, Schütte UME, Beck DL, Abdo Z, Fox LK, Williams JE, McGuire MK, and McGuire MA. 2011. Characterization of the Diversity and Temporal Stability of Bacterial Communities in Human Milk. PLoS ONE 6:e21313. 10.1371/journal.pone.0021313

Inform Physiotherapy. 2021. Lactation for Health Professionals August 2021. Available at https://www.registernow.com.au/secure/Register.aspx? E=43884 (accessed 24 June 2021).

Ingman WV, Glynn DJ, and Hutchinson MR. 2014. Inflammatory mediators in mastitis and lactation insufficiency. J Mammary Gland Biol Neoplasia 19:161-167. 10.1007/s10911014-9325-9

Jahanfar S, Ng CJ, and Teng CL. 2013. Antibiotics for mastitis in breastfeeding women. Cochrane Database of Systematic Reviews:CD005458. 10.1002/14651858.CD005458.pub3

Karcioglu O, Topacoglu H, Dikme O, and Dikme O. 2018. A systematic review of the pain scales in adults: Which to use? Am J Emerg Med 36:707-714. 10.1016/j.ajem.2018.01.008

Kvist LJ. 2006. Care and treatment of women with inflammatory symptoms of the breast during lactation (Doctoral thesis). Fakulteten för samhälls-och livsvetenskaper, Karlstad University.

Kvist LJ, Larsson BW, Hall-Lord ML, Steen A, and Schalen C. 2008. The role of bacteria in lactational mastitis and some considerations of the use of antibiotic treatment. International Breastfeeding Journal 3:6. 10.1186/1746-4358-3-6

Kyte DG, Calvert M, van der Wees PJ, ten Hove R, Tolan S, and Hill JC. 2014. An introduction to patient-reported outcome measures (PROMs) in physiotherapy. Physiotherapy 101:119-125. 10.1016/j.physio.2014.11.003

LactaResearch Group. 2018. LactaPedia: Mastitis. In: Boss M, and Hartmann P, editors. Frauenfeld (CH): Family Larsson-Rosenquist Foundation.

LaTuga MS, Stuebe A, and Seed PC. 2014. A Review of the Source and Function of Microbiota in Breast Milk. Semin Reprod Med 32:068-073.

Maccallum RC, Widaman KF, Zhang S, and Hong S. 1999. Sample Size in Factor Analysis. Psychological Methods 4:84-99. 10.1037/1082-989X.4.1.84

Marks GN, McMillan J, Jones FL, and Ainley J. 2000. The measurement of socioeconomic status for the reporting of nationally comparable outcomes of schooling. Draft Report by the National Education Performance Monitoring Taskforce, Australian Council for Educational Research and Sociology Program, Research School of Social Sciences, Australian National University, www mceecdya edu au/vervel_resources/socioeconomicstatus_file pdf.

R Core Team. 2017. R: A language and environment for statistical computing. Vienna, Austria: R Foundation for Statistical Computing.

REDCap. 2004. Citations. Available at https://projectredcap.org/resources/citations/.

Scott A, Khan K, Cook J, and Duronio V. 2004. What is "inflammation"? Are we ready to move beyond Celsus? British journal of sports medicine 38:248-249.

StataCorp. 2017. Stata Statistical Software: Release 15. College Station, TX: StataCorp LLC. 
390

391

392

393

394

395

396

397

398

399

400

401

402
Streiner Da. 2015. Health measurement scales : a practical guide to their development and use / David L Streiner, Geoffrey R. Norman and John Cairney: New York, New York : Oxford University Press.

Taber KS. 2018. The Use of Cronbach's Alpha When Developing and Reporting Research Instruments in Science Education. Research in Science Education 48:1273-1296. 10.1007/s11165-016-9602-2

Thompson B. 2004. Chapter 2: Foundational Concepts: Sample Size Considerations in EFA. Exploratory and confirmatory factor analysis: Understanding concepts and applications. Washington, DC, US: American Psychological Association, 24.

Wilson E, Woodd SL, and Benova L. 2020. Incidence of and Risk Factors for Lactational Mastitis: A Systematic Review. J Hum Lact:890334420907898.

$10.1177 / 0890334420907898$ 


\section{Table $\mathbf{1}$ (on next page)}

Breast Inflammatory Symptom Severity Index (BISSI) assessment items

Note: In the BISSI there are seven questions, two of which have sub-questions** ${ }^{*}$. In this paper we consider all sub-questions as an item. Thus, there were 11 items from seven questions of which one question* had two sub-questions and another question" had four sub-questions. The remaining five were stand-alone questions. 
1 Table 1:

2 Breast Inflammatory Symptom Severity Index (BISSI) assessment items

\begin{tabular}{|c|c|c|}
\hline BISSI items & Scale & Inflammatory symptom \\
\hline \multicolumn{3}{|l|}{ 1. Pain* } \\
\hline a) Awareness & 11-point NRS scale & Breast pain (on awareness) \\
\hline b) Touch & 11-point NRS scale & Breast pain (on touch) \\
\hline \multicolumn{3}{|l|}{ 2. Wellness / Sickness ${ }^{\#}$} \\
\hline a) Fever & 11-point NRS scale & Systemic symptoms \\
\hline b) Generalised aches \& pains & 11-point NRS scale & Systemic symptoms \\
\hline c) Headache & 11-point NRS scale & Systemic symptoms \\
\hline d) Sickness (unspecified) & 11-point NRS scale & Systemic symptoms \\
\hline 3. Hardness / Tightness & 5-point Likert scale & Breast swelling due to ICLB \\
\hline 4. Temperature of affected area & 5-point Likert scale & Breast heat due to ICLB \\
\hline 5. Redness & 5-point Likert scale & Breast redness due to ICLB \\
\hline 6. Affected Area & 5-point Likert scale & Size of affected breast area \\
\hline 7. Impact & 11-point NRS scale & Functional loss \\
\hline \multicolumn{3}{|l|}{ Total score: } \\
\hline Awareness & Maximum of 80 & \\
\hline Touch & Maximum of 80 & \\
\hline
\end{tabular}




\section{Table 2 (on next page)}

Mothers' demographics $(N=160)$

$Q=$ Quartile. ${ }^{*} n=2$ maternal date of births not reported. ${ }^{\wedge} n=7$ not reported. ${ }^{\circledR} n=2$ not reported. ${ }^{\#} n=33$ not reported. ${ }^{+} n=8$ not reported. ' Most disadvantaged. ' Most advantaged. ${ }^{\$}$ Occupational socioeconomic status scale - modified version of Marks (2000) (Marks et al. 2000) : Group 1 - Senior management and qualified professionals; Group 2 - Other managers and associate professionals; Group 3 - Trades people and skilled staff; Group 4 - Assistants and labourers; Group 5 - Not currently in paid work; Group 6 - Not reported (see Table S1).

${ }^{\star}$ Any indication in the initial clinic notes (excluding BISSI scores), that the mother had systemic symptoms for this ICLB episode. "At initial appointment, for this ICLB episode. 
1 Table 2:

2 Mothers' demographics $(\mathbf{N}=\mathbf{1 6 0})$

\begin{tabular}{|c|c|}
\hline Demographic variable & Median (Q1, Q3) or n (\%) \\
\hline Maternal age* (years) & $35(31,37)$ \\
\hline Range & $25-42$ \\
\hline \multicolumn{2}{|l|}{ Maternal parity $^{\wedge}$} \\
\hline Primiparity & $85(53.1)$ \\
\hline Multiparity@ & $68(42.5)$ \\
\hline Two children & $58(36.3)$ \\
\hline Three children & $8(5)$ \\
\hline Singleton birth & $160(100)$ \\
\hline \multicolumn{2}{|l|}{ Mode of delivery } \\
\hline Vaginal & $90(56.3)$ \\
\hline Caesarean & $37(23.1)$ \\
\hline \multicolumn{2}{|l|}{ Socioeconomic status } \\
\hline \multicolumn{2}{|l|}{ Postal area index ${ }^{+}$} \\
\hline $1^{>}$ & $0(0)$ \\
\hline 2 & $5(3.1)$ \\
\hline 3 & $4(2.5)$ \\
\hline 4 & $11(6.9)$ \\
\hline 5 & $2(1.3)$ \\
\hline 6 & $2(1.3)$ \\
\hline 7 & $26(16.3)$ \\
\hline 8 & $6(3.8)$ \\
\hline 9 & $37(23.1)$ \\
\hline $10^{<}$ & $59(36.9)$ \\
\hline \multicolumn{2}{|c|}{ Private health insurance } \\
\hline Yes & $111(69.4)$ \\
\hline Unknown & $49(30.6)$ \\
\hline \multicolumn{2}{|l|}{ Occupation grouping\$ } \\
\hline 1 & $65(40.6)$ \\
\hline 2 & $60(37.5)$ \\
\hline 3 & $11(6.9)$ \\
\hline 4 & $0(0)$ \\
\hline 5 & $7(4.4)$ \\
\hline 6 & $17(10.6)$ \\
\hline \multicolumn{2}{|l|}{ Month of presentation } \\
\hline Jan & $12(7.5)$ \\
\hline Feb & $10(6.3)$ \\
\hline Mar & $20(12.5)$ \\
\hline Apr & $8(5)$ \\
\hline May & $14(8.8)$ \\
\hline Jun & $11(6.9)$ \\
\hline Jul & $14(8.8)$ \\
\hline Aug & $22(13.8)$ \\
\hline
\end{tabular}




\begin{tabular}{ll}
\hline Sept & $18(11.3)$ \\
Oct & $10(6.3)$ \\
Nov & $14(8.8)$ \\
Dec & $7(4.4)$ \\
Affected breast & $67(41.9)$ \\
Left & $73(45.6)$ \\
Right & $20(12.5)$ \\
Bilateral & $9.5(4.0,21.7)$ \\
Infant age (weeks) & $0.57-91.25$ \\
Range & $2(1,3)$ \\
Symptom onset (days ago) & \\
Episode systemic symptoms ${ }^{\&}$ & $84(52.5)$ \\
Yes & $21(13.1)$ \\
No & $55(34.4)$ \\
Not recorded & \\
Mastitis & $79(49.4)$ \\
Episode & $44(27.5)$ \\
$\quad$ Yes & $37(23.1)$ \\
No & \\
Unable to determine & $54(33.8)$ \\
Initial appointment & $56(35.0)$ \\
Yes & $50(31.3)$ \\
No & \\
Unable to determine & $48(45)$ \\
Antibiotic use & \\
Yes & \\
No & \\
Not recorded & \\
\hline & \\
\hline
\end{tabular}

$3=$ Quartile. ${ }^{*} n=2$ maternal date of births not reported. ${ }^{\wedge} n=7$ not reported. ${ }^{\circledR} n=2$ not reported. ${ }^{*} n=33$ not 4 reported. ${ }^{+} n=8$ not reported. ${ }^{>}$Most disadvantaged. ${ }^{<}$Most advantaged. ${ }^{\$}$ Occupational socioeconomic status scale5 modified version of Marks (2000) (Marks et al. 2000): Group 1 -Senior management and qualified professionals;

6 Group 2 -Other managers and associate professionals; Group 3 - Trades people and skilled staff; Group 4 7 Assistants and labourers; Group 5 - Not currently in paid work; Group 6-Not reported (see Table S1). \& Any 8 indication in the initial clinic notes (excluding BISSI scores), that the mother had systemic symptoms for this ICLB

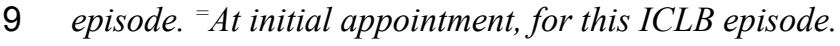




\section{Table 3(on next page)}

Factor loading of BISSI for the extracted factors through principal component analysis $(n=160)$

Extraction Method: Principal Component. Rotation Method: Varimax with Kaiser Normalization. ${ }^{*} P C A A=$ Physical characteristics of affected area. 
1 Table 3:

2 Factor loading of BISSI for the extracted factors through principal component analysis

$3 \quad(\mathbf{n}=\mathbf{1 6 0})$

\begin{tabular}{llcccc}
\hline \multirow{2}{*}{$\begin{array}{l}\text { Item } \\
\text { number }\end{array}$} & Factor & Item description & \multicolumn{3}{c}{$\begin{array}{c}\text { Extracted factors } \\
\text { (correlations) }\end{array}$} \\
\cline { 2 - 5 } & & 1 & 2 & 3 & 4 \\
\hline
\end{tabular}

$\begin{array}{llll}1 & \text { Wellness } & \text { Headache } & 0.727 \\ 2 & \text { Wellness } & \text { Ache } & 0.806 \\ 3 & \text { Wellness } & \text { Fever } & 0.838\end{array}$

4 Pain

Touch 0.856

5 Pain Awareness 0.892

6 PCAA* Impact 0.675

$7 \quad$ PCAA* $^{*} \quad$ Affected area $\quad 0.720$

$8 \quad$ PCAA* Hardness 0.765

9 Inflammation Temperature $\quad 0.833$

10 Inflammation Redness $\quad 0.861$

$\begin{array}{lllll}\text { Eigenvalues of Factors } \quad 3.255 & 1.554 & 1.208 & 1.103\end{array}$

$\begin{array}{lllll}\% \text { variance explained by } \quad 32.552 & 15.54 & 12.082 & 11.028\end{array}$ factors

4 Extraction Method: Principal Component. Rotation Method: Varimax with Kaiser Normalization.

$5 * P C A A=$ Physical characteristics of affected area. 
Table 4 (on next page)

Differences in the BISSI item scores and total score between mothers with high or low symptom severity

*PCAA= Physical characteristics of affected area 
1 Table 4:

2 Differences in the BISSI item scores and total score between mothers with high or low

3 symptom severity

\begin{tabular}{lccccc}
\hline \multirow{2}{*}{ Factors } & \multicolumn{2}{c}{ Low } & \multicolumn{2}{c}{ High } & \multirow{2}{*}{ P Value } \\
\cline { 2 - 5 } & Mean & SD & Mean & SD & \\
Wellness & 1.7 & 4.7 & 7.4 & 7.0 & 0.000002 \\
Pain & 5.3 & 3.7 & 8.6 & 4.7 & 0.000069 \\
PCAA* & 18.1 & 5.0 & 20.7 & 4.5 & 0.006078 \\
Inflammation & 7.8 & 2.8 & 12.7 & 4.1 & $<0.000001$ \\
Total score & 32.9 & 10.3 & 49.4 & 13.2 & $<0.000001$ \\
\hline
\end{tabular}




\section{Table 5 (on next page)}

Area under the Curve (AUC) analysis of mothers with high symptom severity

*PCAA = Physical characteristics of affected area 
1 Table 5:

2 Area under the Curve (AUC) analysis of mothers with high symptom severity

\begin{tabular}{lrrrc}
\hline \multirow{2}{*}{ Factors } & \multirow{2}{*}{ AUC } & \multirow{2}{*}{ P Value } & \multicolumn{2}{c}{ 95\% CI } \\
\cline { 4 - 5 } Wellness & .770 & .000001 & 0.679449 & 0.860895 \\
Pain & .704 & .000222 & 0.607184 & 0.801216 \\
PCAA* & .648 & .007592 & 0.543919 & 0.751386 \\
Inflammation & .832 & .000001 & 0.756933 & 0.906758 \\
BISSI & .857 & .000001 & 0.784782 & 0.929503 \\
\hline
\end{tabular}

3

*PCAA $=$ Physical characteristics of affected area

4 
Table 6(on next page)

Internal consistency analysis of BISSI and the proposed factors

$* P C A A=$ Physical characteristics of affected area 
1 Table 6:

2 Internal consistency analysis of BISSI and the proposed factors

\begin{tabular}{lcccc}
\hline Factor & Items numbers & $\begin{array}{c}\text { Descriptive } \\
\text { statistics } \\
\text { Mean (sd) }\end{array}$ & $\begin{array}{c}\text { Cronbach's } \\
\text { alpha }\end{array}$ & $\begin{array}{c}\text { Factor total } \\
\text { correlation } \\
\text { coefficient }\end{array}$ \\
\hline Total score & $1-10$ & $40.1(13.3)$ & 0.742 & - \\
Wellness & $1-3$ & $3.7(5.9)$ & 0.754 & 0.743 \\
Pain & 4,5 & $6.6(4.3)$ & 0.784 & 0.675 \\
PCAA* & $6-8$ & $19.1(4.9)$ & 0.586 & 0.681 \\
Inflammation & 9,10 & $10.1(4.1)$ & 0.720 & 0.611 \\
\hline *PAA= Physical characteristics of affected area & &
\end{tabular}

4 
Table 7 (on next page)

Internal consistency analysis of BISSI items 
1 Table 7:

2 Internal consistency analysis of BISSI items

\begin{tabular}{clcccc}
\hline $\begin{array}{c}\text { Item } \\
\text { Number }\end{array}$ & Item description & $\begin{array}{c}\text { Item } \\
\text { Mean (SD) }\end{array}$ & $\begin{array}{c}\text { BISSI Mean } \\
\text { if Item } \\
\text { Deleted }\end{array}$ & $\begin{array}{c}\text { Corrected } \\
\text { Item-Total } \\
\text { Correlation }\end{array}$ & $\begin{array}{c}\text { Cronbach's } \\
\text { Alpha if Item } \\
\text { Deleted }\end{array}$ \\
\hline 1 & Fever & $0.8(2.1)$ & 38.8 & 0.35 & 0.753 \\
2 & Aches & $1.4(1.4)$ & 38.1 & 0.56 & 0.725 \\
3 & Headache & $1.5(1.5)$ & 38.0 & 0.50 & 0.732 \\
4 & Pain Awareness & $2.1(2.1)$ & 37.5 & 0.44 & 0.742 \\
5 & Pain Touch & $4.6(2.6)$ & 35.0 & 0.52 & 0.730 \\
6 & Hardness & $4.9(2.3)$ & 34.6 & 0.37 & 0.751 \\
7 & Area & $6.3(1.8)$ & 33.3 & 0.42 & 0.747 \\
8 & Impact & $6.6(2.5)$ & 33.0 & 0.44 & 0.742 \\
9 & Redness & $6.2(2.3)$ & 33.4 & 0.25 & 0.768 \\
10 & Temperature & $5.1(2.3)$ & 34.4 & 0.42 & 0.744 \\
\hline
\end{tabular}

3 


\section{Figure 1}

\section{Eligibility}

Figure 1:

Eligibility

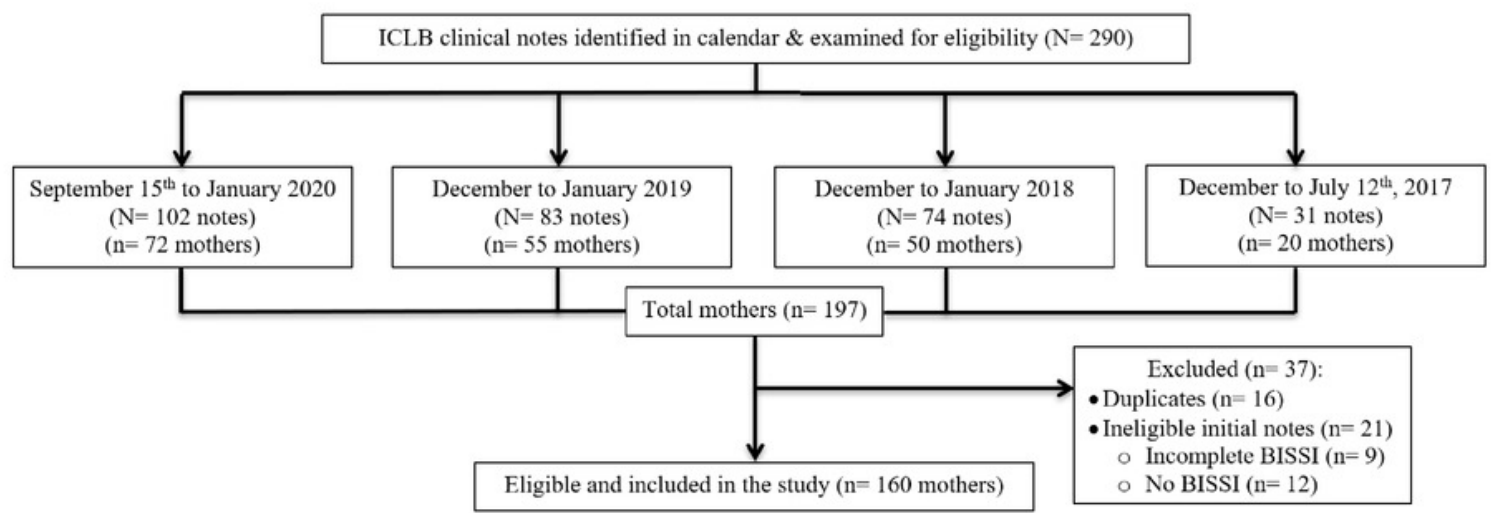


Figure 2

\section{Affected breast quadrants}

Figure 2:

Affected breast quadrants

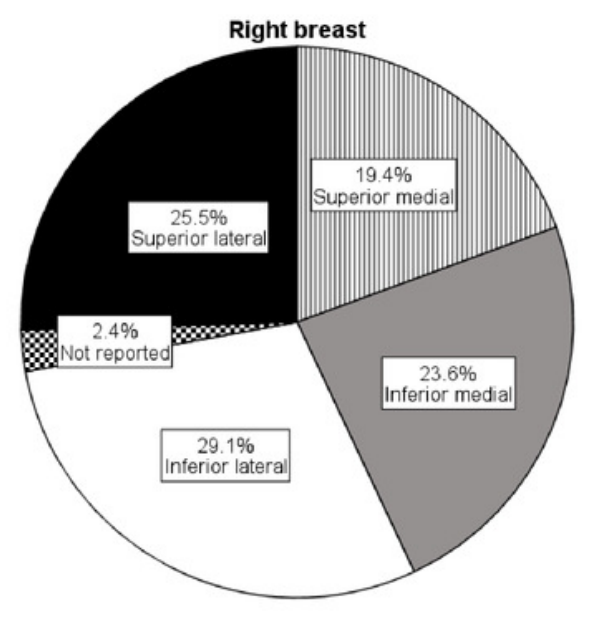

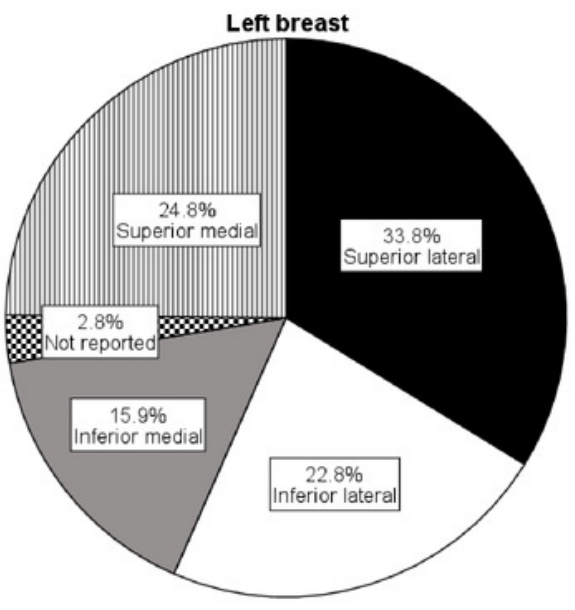


Figure 3

Bar chart illustrating factor loading 
Figure 3:

Bar chart illustrating factor loading

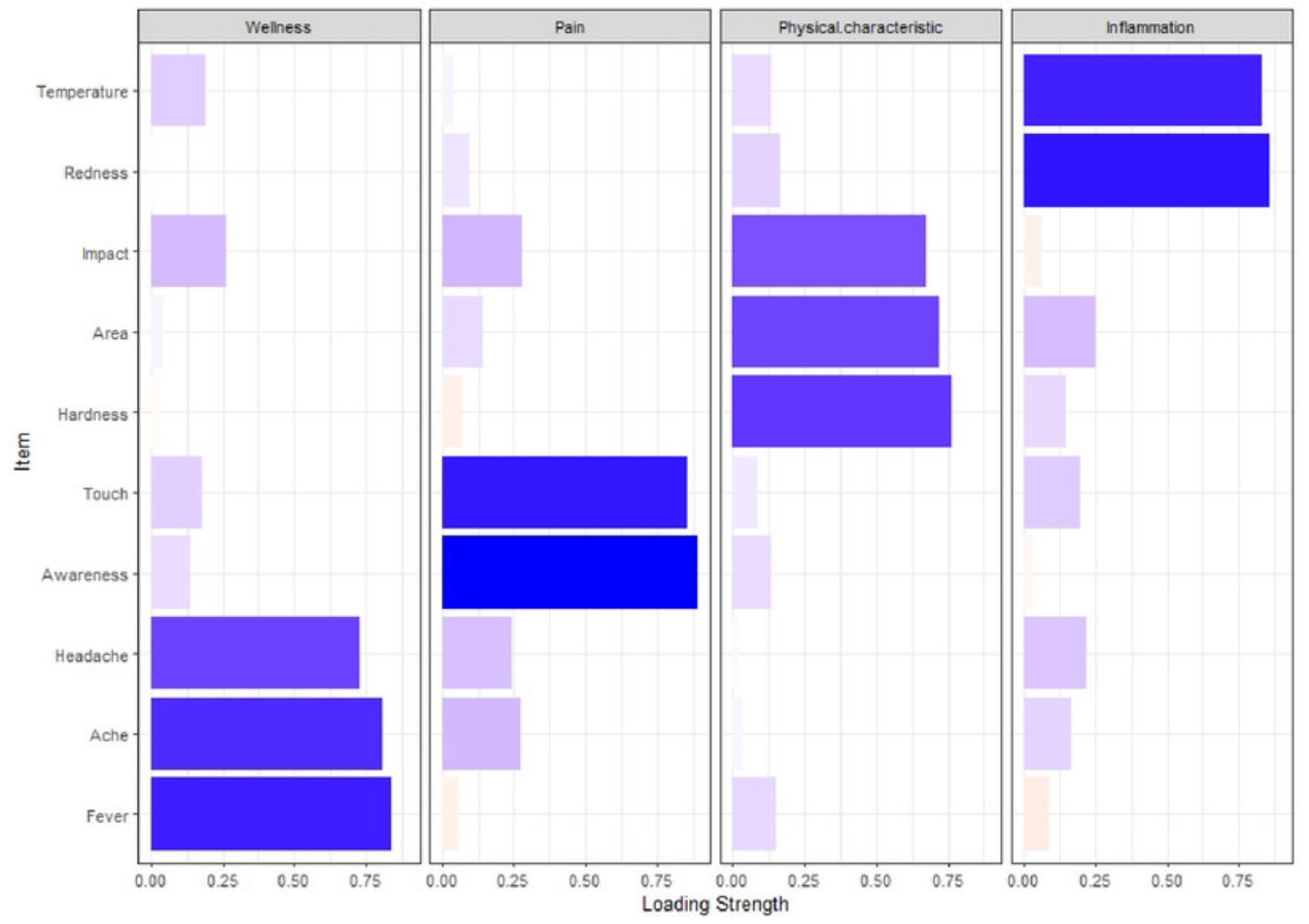


Figure 4

ROC analysis of high symptom severity 
Figure 4:

ROC analysis of high symptom severity
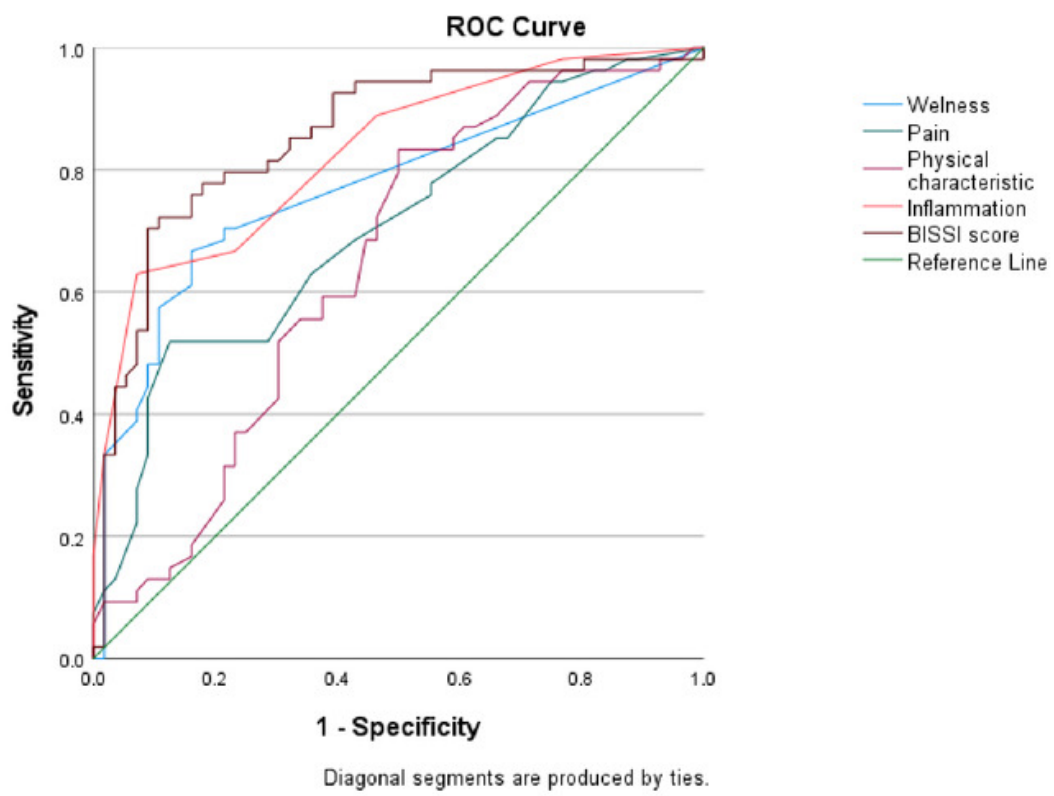